\title{
Review of nutritional status and dietetic interventions in patients suffering from upper-gastrointestinal cancers
}

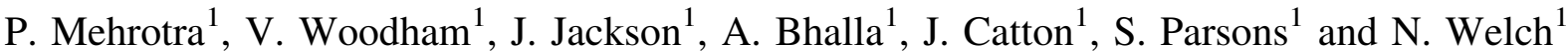 \\ ${ }^{1}$ Department of General Surgery, Nottingham City Hospital, Hucknall Road, Nottingham NG5 1LB, UK
}

Patients with oesophageal and gastric cancers often present with weight loss and poor nutrition ${ }^{(1)}$. Nutritional status is a predictive factor in post-operative outcomes ${ }^{(2)}$. Neo-adjuvant chemotherapy followed by surgery is the treatment of choice for the majority of these patients ${ }^{(3)}$. The effects of chemotherapy on weight loss and nutrition is of clinical importance ${ }^{(4)}$.

A prospective cohort of patients undergoing neo-adjuvant chemotherapy prior to surgical resection for oesophageal and gastric cancers within a major teaching hospital were assessed. Primary end points included weight change during neo-adjuvant chemotherapy and extent of dietetic intervention.

Fifty-two patients were assessed. Sixty-seven percent $(n$ 34) of patients were noted to have weight loss between the time of diagnosis and completion of pre-operative chemotherapy. $21 \%(n 11)$ of patients gained weight, the average percentage weight gain being $5 \%$. The average percentage weight loss was $6 \%(+13 \%$ to $-22 \%)$. Thirteen percent $(n 7)$ of patients suffered severe weight loss $(>10 \%$ total body weight). Eighteen percent $(n 10)$ of patients received dietetic intervention which included oral nutritional supplements $(n 4)$, nasogastric supplements $\left(\begin{array}{l}n \\ 2\end{array}\right)$ and nasojejunal supplements $(n 4)$. Of the patients that gained weight, only $18 \%(n 2)$ received dietetic intervention.

The majority of patients undergoing neo-adjuvant chemotherapy prior to surgery for oesophageal and gastric cancers experienced weight loss during the period of treatment. Early and aggressive nutritional assessment and support may help prevent or reduce preoperative weight loss and improve patient outcomes.

1. Gencer D, Kästle-Larralde N, Pilz LR et al. (2009) Onkologie 32, 380-386.

2. Han-Geurts IJ, Hop WC, Tran TC et al. (2006) Dig Surg 23, 159-163.

3. Mezhir JJ, Tang LH \& Coit DG (2010) J Surg Oncol 101, 305-314.

4. Bower M \& Martin R (2009) J Surg Oncol 100, 82-87. 